\title{
Advanced optical processing systems combining linear pulse shapers and fibre-based nonlinear switches
}

\author{
P. Petropoulos, F. Parmigiani, T.T. Ng, M. Ibsen, D.J. Richardson \\ Optoelectronics Research Centre, University of Southampton, Southampton SO17 1BJ, United Kingdom. \\ Tel: +44 2380592014, Fax: +44 2380593149, Email: pp@orc.soton.ac.uk
}

\begin{abstract}
The combination of linear pulse shaping, achieved using superstructured fibre Bragg gratings, and fibre-based nonlinear switching facilitates advanced all-optical processing of signals. Some applications include suppression of timing jitter, and demultiplexing based on frequency-shifting of OTDM signals.
\end{abstract}

Keywords: optical communications, pulse shaping, optical processing, fibre Bragg gratings, Kerr nonlinearities

\section{Introduction}

Fibre-based optical signal processing has progressed to a degree that sophisticated custom-made solutions based on a combination of technologies can be envisaged to address a variety of processing applications. Highly customisable spectral filters can be implemented using fibre Bragg grating technology, offering the route to low loss, polarisation insensitive devices which can be readily integrated to fibre systems, and can be inexpensive in their fabrication. Advances in fibre fabrication on the other hand, allow the implementation of fibre waveguides with large values of nonlinearity and highly customisable dispersion characteristics, allowing the implementation of nonlinear switching devices with exceptional performance operating at reasonable power levels.

This talk will review some application examples which combine pulse shaping in fibre Bragg gratings with highly nonlinear fibre (HNLF)-based optical switches.

\section{Pulse shaping in superstructured fibre Bragg gratings}

Fibre Bragg gratings have found widespread application in telecommunication systems, in such areas as add-drop multiplexing of WDM channels and dispersion compensation. Common apodised grating designs exhibit an almost flat spectral response with sharp cut-off edges. However, superstructured fibre Bragg gratings (SSFBGs), which are a more sophisticated class of fibre gratings in terms of design and fabrication, allow for the implementation of filters with almost arbitrary frequency response, thereby enabling applications that require precise control over the temporal and spectral characteristics of optical waveforms. The distinguishing characteristic of SSFBGs is that they contain a relatively slowly varying spatial modulation envelope upon the rapidly varying refractive index profile of the grating. This can be either an amplitude or a phase envelope, depending on the response sought for. Fabrication of SSFBGs can be achieved using phase masks specially designed for the purpose, but a much more economic and flexible solution relies on a 'plane-by-plane' grating writing technique, which allows superstructures of even a high degree of complexity to be implemented using only a uniform phase mask. The 'plane-by-plane' writing technique has been employed for the fabrication of all SSFBGs reported in this work.

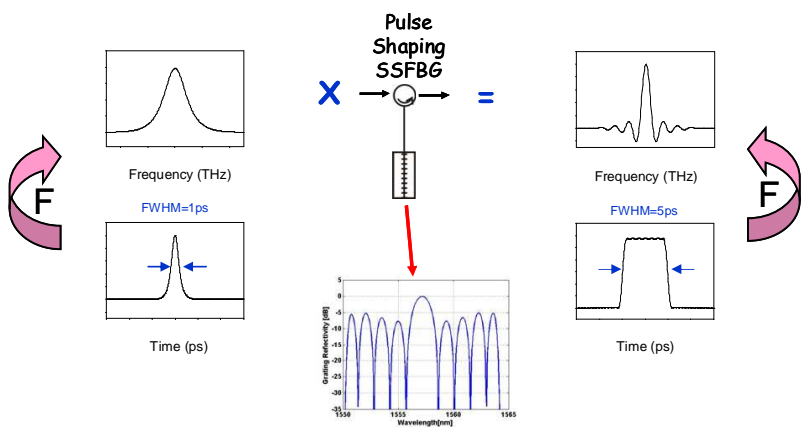

Figure 1 : SSFBGs for linear pulse shaping applications

Once the capability to develop filters with custom-made frequency responses is available, it is then possible to use these filters to shape the spectral (hence, temporal as well) characteristics of pre-known pulses into other - potentially more useful - waveforms. This process is shown in an example in Fig.1, where an SSFBG is used to shape short $\mathrm{sech}^{2}$ pulses into rectangular waveforms. The Figure also shows the spectral response of the SSFBG required for this function.

The generation of rectangular [1], parabolic [2] or even saw-tooth shaped [3] ps-long pulses using this technique has already been demonstrated.

\section{Nonlinear processing systems incorporating pulse shaping elements}

The capability to tailor the shapes of short optical pulses can greatly enhance the performance of nonlinear processing systems, and in several occasions can give rise to new applications that would not be possible otherwise. A generic diagram showing the configuration of a system that incorporates a combination of linear shaping elements and a nonlinear switch is shown in Fig.2. The shaping elements (i.e. pulse shaping SSFBGs in our applications) can be used to control the shape of either of the control signal of the switch, the data signal, the switch output, or a combination of these. It is recognised however, that in most applications it is the control signal which is pre-shaped; this is both because this is usually the most crucial signal for the operation of the switch, and also because this is typically a locally generated signal with well-controlled characteristics. 


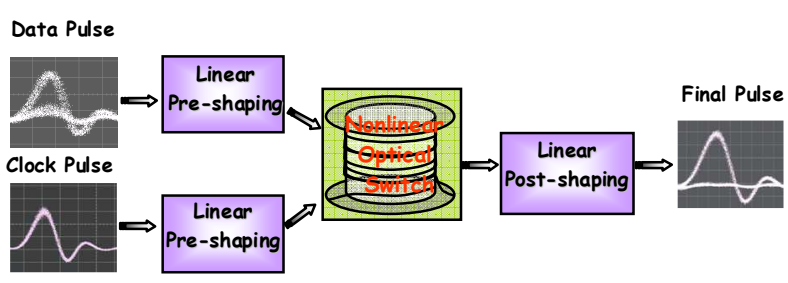

Figure 2 : Generic block diagram of a processing system incorporating both linear and nonlinear elements

In order to appreciate more easily the significance of pulse shaping in these applications, we will investigate a simple example which studies the nonlinear propagation of two different pulse shapes in the same length of HNLF [3]. The fibre has a nonlinear coefficient of $19 \mathrm{~W}^{-1} \mathrm{~km}^{-1}$, dispersion of $-0.31 \mathrm{ps} / \mathrm{nm} / \mathrm{km}$ at $1550 \mathrm{~nm}$, and its length is $310 \mathrm{~m}$. In the first instance the pulses have a Gaussian shape and a full width at half maximum (FWHM) of 10 ps (Fig.3a). The pulse spectrum is recorded at the output of the HNLF with an optical spectrum analyser (Fig.3b). A broadened spectrum exhibiting the well studied characteristics of self-phase modulation (SPM) is clearly observed. In the second case, short (1.3 ps FWHM) Gaussian pulses are shaped into saw-tooth pulses of 10 ps FWHM (see Fig.3a) using a suitably designed SSFBG. The asymmetry in the temporal profile of the saw-tooth pulses gives rise to distinct spectral features on either side of the central wavelength (Fig.3b) see discussion in Ref.[3]. The sharp trailing edge of the pulses gives rise to a broad range of new frequencies extending on the short-wavelength side of the spectrum. In contrast, the long leading edge of the pulses yields a narrower SPM spectrum, which can be observed on the long-wavelength side. Furthermore, all the SPM-generated frequencies on these longer wavelengths are confined within a very small range, owing to the linear variation of intensity profile of the leading pulse edge with time.

This behaviour can be quite significant when used in the context of cross-phase modulation, for add-drop applications of optical time division multiplexed signals [4].

\section{Conclusion}

This paper reviews the benefits that can be gained by combining SSFBG-based pulse shapers operating in a linear fashion with nonlinear switching devices based on HNLFs. These advanced processing systems can be used in the implementation of such applications as signal regeneration, wavelength conversion and demultiplexing to name but a few. Some application examples will be presented in the conference presentation.

\section{Acknowledgments}

The authors acknowledge Furukawa Electric Co. for the loan of the HNLF used in these experiments. The work described herein was supported by the European Union through the FP6 STREP project TRIUMPH (IST-027638 STP) and FP7 Network of Excellence BONE (FP7-ICT-2007-1 216863). (a)

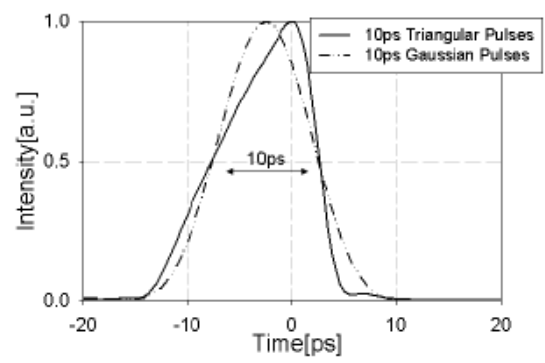

(b)

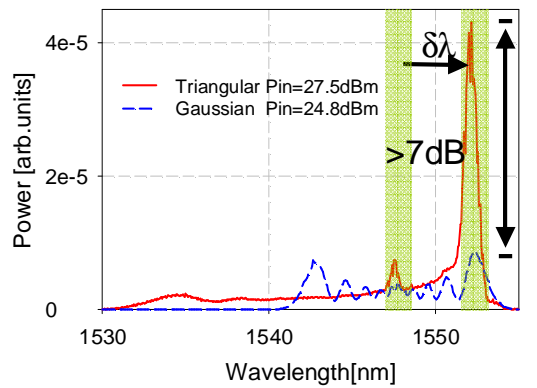

Figure 3 : (a) Comparison between the 10 ps Gaussian and saw-tooth pulse profiles; (b) comparison between the SPM-spectral broadening of Gaussian and saw-tooth pulses.

\section{References}

[1] P. Petropoulos, M. Ibsen, A. D. Ellis, and D. J. Richardson, "Rectangular pulse generation based on pulse reshaping using a superstructured fiber Bragg grating," Journal of Lightwave Technology, vol. 19, pp. 746-752, 2001

[2] F. Parmigiani, P. Petropoulos, M. Ibsen, and D. J. Richardson, "Pulse Retiming Based on XPM Using Parabolic Pulses Formed in a Fiber Bragg Grating," IEEE Photonics Technology Letters, vol. 18, pp. 829-31, 2006.

[3] F. Parmigiani, M. Ibsen, T.T. Ng, L. Provost, P. Petropoulos, D.J. Richardson, "An efficient wavelength converter exploiting a grating based saw-tooth pulse shaper," IEEE Photonics Technology Letters, 2008, to appear.

[4] F. Parmigiani, M. Ibsen, T.T. Ng, P. Petropoulos, D.J. Richardson, "Timing jitter tolerant TDM Demuliplexing using a Saw-Tooth Pulse Shaper," ECOC 2008, 21-25 September 2008, Brussels, Belgium, to be presented. 